\title{
Ética y política: dimensiones prácticas de la experiencia del lenguaje en el filosofar wittgensteiniano
}

\author{
Samuel M. Cabanchik*
}

\begin{abstract}
Resumen: Como suele ocurrir con los clásicos, también la determinación de la orientación dominante de la filosofía de Wittgenstein sigue siendo materia de controversia. En el presente artículo sostengo el papel estratégico de la dimensión ética, como clave de lectura de su pensamiento. El eje fundamental de esta clave deriva de lo que llamaremos "experiencia del lenguaje", tal como se da cuenta de la misma desde el primer al último período de su obra. Se sostendrá que toda la obra de Wittgenstein puede leerse como un campo de experimentación para realizar la experiencia del lenguaje. En el primer período, la experiencia del lenguaje se realiza en la articulación entre el sentido y el sinsentido, a través de la distinción decir/mostrar como punto de apoyo para el acto ético; con posterioridad al Tractatus, son los fenómenos del "ver como" o "notar un aspecto", los que hacen posible el montaje de experimentos lingüísticos en los que la experiencia del lenguaje es realizada. También en la perspectiva de las Investigaciones la experiencia del lenguaje es ocasión para la realización del acto ético, pero ya no en una perspectiva solipsista como la del Tractatus, sino en una comunitaria y, en ese sentido, quizá política, en una acepción amplia de la palabra.
\end{abstract}

Palabras clave: experiencia, lenguaje, ética, notar un aspecto

Abstract: As is often the case with the classics, the determination of the dominant orientation of Wittgenstein's philosophy remains a matter of controversy. In this paper, I argue for the recognition of the strategic role of the ethical dimension as a key to the reading of his thought. This key has its center in what we call "the experience of language", a dimension that can be found from the first to the last period of his work. I will argue that Wittgenstein's work as a whole can be read as an investigation on the nature of the experience of language. In the first period, the experience of language is

\footnotetext{
* Doctor en Filosofía por la Universidad de Buenos Aires, en donde es profesor de Filosofía Contemporánea y Fundamentos de Filosofía. Investigador del CONICET. Ha publicado catorce libros y numerosos artículos y ensayos en medios nacionales e internacionales. Entre sus últimos libros publicados se mencionan: El poema ha sido escrito (2010), Wittgenstein (2010), Figuraciones de la comunidad (coeditor junto a A. Boverio 2014), Desde el Palacio (2015) y Alejandro Korn (2016). Dirección electrónica: samuel.cabanchik@gmail.com
} 
found in the articulation between sense and nonsense. This distinction, articulated with the distinction saying/showing is as a point of support for the ethical act. In the Philosophical Investigations, the experiments that allows analyzing the experience of language are concerned with the phenomena of "seeing as" or "noticing an aspect". Also in this case, the experience of language is linked with the realization of the ethical act, but no longer in a solipsist perspective as in the Tractatus, but in a communitarian way and, in that sense, in a way that is also political, in a broad sense of the word.

Keywords: experience, language, ethics, noticing an aspect

\section{Introducción}

Sartre afirmó que una filosofía debe ser al mismo tiempo "totalización del saber, método, Idea reguladora, arma ofensiva y comunidad de lenguaje". ${ }^{1}$ Sostuvo que tantas exigencias convergentes hacen raras, escasas a las épocas de auténtica creación filosófica. Mientras mantiene su vigor, una filosofía es para Sartre el horizonte cultural insuperable de una época.

Esta concepción de la filosofía exagera en dos sentidos: pide demasiado y se lo pide todo a una filosofía. En estos términos, la polémica se hace inevitable: ¿es posible que una y sólo una filosofía se constituya en el horizonte cultural insuperable de toda una época? Como digo, hay en este concepto más de una exageración. Sin embargo, convenientemente corregido puede ser reformulado así: "una filosofía está viva mientras integre, estratégicamente, un horizonte cultural para toda una corriente de pensamiento en una época dada". En este sentido, argüiré que la filosofía de Ludwig Wittgenstein está viva.

Para que esa vitalidad se realice, la filosofía en cuestión debe hallarse abierta para sí misma, a través de las diversas apropiaciones a las que da lugar $y$, en este sentido, toda ampliación de sus horizontes requiere una problematización con su propia "metafilosofía"-

En ese proceso de apropiación encontramos dos movimientos superpuestos: 1. nuevas preguntas encuentran respuesta en sus proposiciones, que al responderlas se vuelven otras; 2. aquellas cuestiones que aún se agitan

\footnotetext{
${ }^{1}$ Sartre, Jean-Paul, Crítica de la rąón dialéctica, Buenos Aires, Losada, 1963, p. 17.
} 
en su texto y que quedaron sin respuesta a la espera de nuevas claves, encuentran su concreción en el marco de nuevos paisajes conceptuales.

Durante el siglo XX, la obra de Wittgenstein impactó sobre el trabajo que estaban realizando algunos de los principales referentes de la llamada "filosofía analítica": en el primer período de su filosofía, fue el positivismo lógico del Círculo de Viena quien recibió su influjo; en su segundo período, la llamada filosofía del lenguaje ordinario.

Posteriormente, su discusión de la posibilidad de concebir un lenguaje privado dio lugar a numerosos debates, hasta inspirar la influyente relectura que, de las Investigaciones filosóficas, hiciera Saúl Kripke, ${ }^{2}$ la que a su vez abrió intensas controversias interpretativas. ${ }^{3}$ Paralelamente, la llamada "filosofía continental" -en contraste con "los analíticos"- fue interesándose más y más en los aspectos prácticos de su pensamiento, dando diversas interpretaciones a su concepción de la ética, la estética y la mística. ${ }^{4}$

En la actualidad asistimos a nuevas apropiaciones de su obra en autores de relevancia en el panorama filosófico continental, como Giorgio Agamben ${ }^{5}$ y Paolo Virno, ${ }^{6}$ e incluso Alain Badiou le ha dedicado un trabajo crítico. ${ }^{7}$

En la extensa bibliografía que contiene la huella wittgensteiniana en el siglo XX, el lector encontrará un corpus notable para el estudio de los principales contenidos, sea que se los desarrolle con fidelidad discipular o a través de la polémica y la crítica. Sin embargo, es probable que poco de todo ello estimule una reorientación interpretativa. Podemos decir que ya hay un Wittgenstein

${ }^{2}$ Kripke, Saul A., Wittgenstein. On Rules and Private Language. An Elementary Exposition, Cambridge (Mass.), Harvard University Press, 1982.

${ }^{3}$ Un excelente panorama se encuentra en Pedro Karczmarczyk, El argumento del lenguaje privado a contrapelo, La Plata, Edulp, 2011.

${ }_{4}$ Para una muy aguda presentación del elemento místico en toda la obra de Wittgenstein, véase Oscar del Barco, El abandono de las palabras, Córdoba, UNC, 1994.

${ }^{5}$ Entre muchos lugares de su obra, ver Agamben, Giorgio, Infancia e historia, Buenos Aires, Adriana Hidalgo editora, 2007.

${ }^{6}$ Entre otros, especialmente Virno, Paolo, Cuando el verbo se hace carne. Lenguaje y naturaleza bumana, Buenos Aires, Cactus y Tinta Limón, 2004.

${ }^{7}$ Badiou, Alain, L'antiphilosophie de Wittgenstein, Paris, Nous, 2009. 
canónico para materias como la filosofía del lenguaje y de la mente y, en menor medida, para las cuestiones estéticas y, en general, prácticas.

Donde en cambio cabe hallar las nuevas preguntas en los dos sentidos aludidos más arriba, es en aquellas tradiciones de pensamiento contemporáneo que, surgidas en tierras muy distantes de las que recibieron la siembra y la cosecha wittgensteinianas, permiten renovar la recepción de su obra, llevándola precisamente más allá de las fronteras de escuela.

Así, por ejemplo, Wittgenstein, que en sus propios términos no fue un pensador político, se vuelve significativo para todo lo que podría reunirse bajo la etiqueta "antropología política", abarcando temas como la dimensión comunitaria de la existencia, los confines de la política o lo impolítico, e incluso mucho de lo que se piensa aun a partir de la expresión "biopolítica".

Todavía es prematuro intentar una sistematización de este nuevo panorama sobre su legado filosófico, como también lo sería pretender una evaluación en términos de corrección, pertinencia o fertilidad. Sólo podemos ofrecer nuestra propia renovación de las preguntas y de las interpretaciones, como una orientación para el recorrido de los horizontes wittgensteinianos que avizoramos.

\section{La experiencia del lenguaje}

\section{El hablante, el lingüista, el filósofo}

Como hablantes de una lengua tenemos la experiencia del lenguaje en el acto participativo de la comunicación -incluso en el diálogo interior-. Este es el fenómeno en bruto al que remitirán en sus respectivos ámbitos tanto el lingüista como el filósofo. Pero el modo de la experiencia participativa está contenido dentro de las subordinaciones de la normatividad de la lengua y de la normalidad de la situación del caso, en las que, contra lo que siente y cree el hablante, quienes mandan son el mundo y la gramática. En la práctica del lenguaje no tomamos al lenguaje como objeto de nuestra experiencia, sino que lo cargamos con nosotros al tener experiencia de los objetos, en sus contenidos y en sus formas. 
Este es el punto de partida que el lingüista y el filósofo han de tener en cuenta como fuente de sus elaboraciones, pero tanto o más para construir sus respectivas distancias que para aprender de él. En efecto, hay una gran diferencia entre el saber práctico que le cabe al hablante en tanto tal y el saber teórico que lo toma como objeto. Pero aquí hay un inmediato contraste, una separación sustantiva entre lo que determina, por una parte, al conocimiento lingüístico, y por otra parte al filosófico.

La lingüística no toma como su objeto al lenguaje en la facultad de su práctica, sino como base empírica de una teoría. Su asunto no será hacer la experiencia del lenguaje como tal. Una referencia esclarecedora para el entendimiento de esta diferenciación de campos y prácticas de saber la encontramos en la obra de Jean-Claude Milner, un lingüista que no se ahorra las consideraciones epistemológicas y filosóficas pertinentes, como cuando establece cuáles son los hechos primitivos de su disciplina, estenografiados en las palabras "lenguaje" y "lengua":

El primero de estos hechos es que hay seres hablantes, que producen formaciones de lenguaje. Llamemos a esto factum loquendi. Supone ya la posibilidad de distinguir una producción de lenguaje de lo que no lo es. (...) $\mathrm{El}$ nombre corriente de este hecho bruto es el lenguaje. (...) Se comprende en qué sentido la lingüística no tiene por objeto al lenguaje; lo toma por axioma $^{8}$.

Y más adelante:

Pero la lingüística no puede quedarse ahí; tiene que admitir algo más que la sola y masiva existencia del lenguaje: admite que los seres humanos hablan lenguas. ${ }^{9}$

Por lo tanto:

La lingüística debe darse como hechos primarios: el hecho de la lengua, el factum linguae, (...) que deberá ser distinguido del factum loquendi (...); el hecho de las lenguas, el factum linguarum, es decir, que sean diversas, pero formando siempre

\footnotetext{
${ }^{8}$ Milner, Jean-Claude, Introducción a una ciencia del lenguaje, Buenos Aires, Manantial, 2000, pp. 41 y sig.

${ }_{9}^{9}$ Ibidem, p. 43.
} 
una clase homogénea (...); el hecho de que las lenguas puedan ser descriptas en términos de propiedades. Este hecho puede recibir un nombre más preciso: hecho de la gramática, factum gramaticae. ${ }^{10}$

Por el contrario, es evidente tanto para Milner como para nosotros, que el punto de partida de la filosofía es el factum loquendi. Ahora bien, según sea la actitud teórica o práctica que adopte la misma, tendremos un resultado muy distinto. En efecto, asumamos que la pregunta filosófica no evite tomar sus formas típicas: ¿por qué hay lenguaje? ¿Cuál es su origen, esencia o fundamento? Y bien, el metafísico o el teórico semántico responden con alguna especulación o alguna teoría; el filósofo crítico, Wittgenstein claramente como veremos, responde: hay lenguaje sin fundamentos ni esencia, como un hecho originario del que sólo cabe hacer la experiencia.

La contraposición entre la lingüística y la obra de Wittgenstein es clara para el propio Milner, quien se refiere a ello en estos términos:

La doctrina del segundo Wittgenstein, por ejemplo, implica negar que una lengua exista de otro modo que como horizonte fantasmático: no hay más que palabras y expresiones particulares, cuyas reglas de empleo es preciso establecer una por una. Estas reglas no se combinan necesariamente y no forman ese todo que podríamos llamar una lengua ${ }^{11}$.

$Y$ en cuanto al conocido recurso wittgensteiniano a la descripción del lenguaje en términos de juegos, agrega en una larga nota:

Es posible que la noción de "juegos de lenguaje" se justifique de este modo: en materia de lenguas naturales sólo se puede hablar de reglas cuando estas reglas están enunciadas de manera explícita, como sucede con las reglas de un juego; ello equivale a tratar tal o cual dato de lengua como un juego, aun sabiéndose que, en su realidad empírica, el fenómeno de lengua no es del orden del juego. Recíprocamente, si no se trata a las lenguas en términos de juego, no se puede hablar de reglas a su respecto. ${ }^{12}$

\footnotetext{
${ }^{10}$ Ibidem, pp. 44 y sig.

${ }^{11}$ Idem.

12 Ibidem, p. 263.
} 
A diferencia de Wittgenstein, que al instrumentar la descripción del lenguaje en términos de juegos se habilita a hablar del problema de la regla y su aplicación, el lingüista, interesado en lograr un conocimiento empírico y corroborable de las lenguas y sus propiedades, rechazará hablar de reglas y de juegos, para reafirmar la existencia de su objeto de experiencia teórica, no práctica: las lenguas, sus gramáticas y sus leyes.

Pero obsérvese que entre el campo práctico-descriptivo en el que se mueve Wittgenstein y el teórico-explicativo en el que lo hace la lingüística, no hay competencia sino más bien indiferencia mutua. En cambio, la filosofía del lenguaje como teoría semántica no puede soslayar ambas internas, pues por un lado compite por el status científico con la lingüística -aunque no está descartado que puedan complementarse-; por otro, denuesta el giro práctico que la gangrena desde sus mismas entrañas, ejemplificado en el filósofo que renuncia a la teoría.

Si nos giramos ahora hacia el olvidado hablante a expensas del cual, finalmente, viven todas estas variantes del saber, vemos el lugar subordinado que le reservan tanto el lingüista como el semántico, frente a la hospitalidad con la que lo alberga el practicante del "experimentum linguae", para usar la feliz expresión con la que el filósofo Giorgio Agamben denomina a la experiencia de lo trascendental en el lenguaje. Porque, en efecto, tanto la distancia como la solidaridad entre la espontaneidad de la participación en el lenguaje y la artificialidad de la experiencia del lenguaje, puede recogerse en lo que tienen de común y de distinto una experiencia, que se manifiesta como primitiva e incalculable, y un experimento, que supone condiciones especiales de experimentación.

Sería arduo y fuera de lugar, pretender reponer en el breve espacio de este ensayo, la compleja urdimbre tejida por Agamben para dar cuenta, a la vez, de las condiciones de posibilidad de ambas experiencias, la originaria del lenguaje para el hablante, y las del experimentum linguae para el filósofo. ${ }^{13}$ Por ello apresuraremos el paso para llegar al núcleo esencial del trascendental en juego. Contra un lugar común aceptado acríticamente, de la mano de Émile Benveniste y su diferenciación entre lo semiótico y lo semántico, o en una terminología más extendida, entre lengua y habla o discurso, Agamben sostiene

${ }^{13}$ Véase Agamben, Giorgio, Infancia e historia, páginas 59 a 86 y 213 a 222. 
que el viviente no humano está desde siempre en la lengua. Es decir, no es que los animales no dispongan de signos, como es obvio sí disponen, sino que no padecen de su ausencia. Por el contrario, el animal humano se caracteriza por poseer in-fancia, esto es, por comenzar su existencia en la condición de un viviente que no habla.

Es entonces por el rodeo de la infancia que se constituye el hablante, al mismo tiempo que se erige en sujeto de experiencias. Al mito de un sujeto y una experiencia dados por fuera del lenguaje, se requiere oponer el concepto del par sujeto-experiencia como constituido dentro del proceso a través del cual un infante deviene hablante. Es en esa suerte de experiencia sin sujeto, si se me permite la expresión, que cobra significación y realidad el hecho de que se habla, (lo que Milner llama el factum loquendi).

Ahora bien, si esta es la condición histórico-trascendental, como dice Agamben, de quien participa del lenguaje, para lo que no se requiere ni su ciencia ni el experimento del filósofo, ¿qué es lo que ganamos con el conocimiento que estas prácticas y campos de saber articulan? En particular, en relación a nuestros intereses en este escrito, ¿qué es lo que se experimenta en el experimentum linguae? La respuesta de Agamben no puede ser más contundente y sugestiva:

Aquello con lo que se hace experiencia en el experimentum linguae no es simplemente una imposibilidad de decir: se trata más bien, de una imposibilidad de hablar a partir de una lengua, por lo tanto, de una experiencia - a través de la demora infantil en la diferencia entre lengua y discurso - de la misma facultad o potencia de hablar. Plantear el problema de lo trascendental quiere decir en última instancia preguntar qué significa "tener una facultad", cuál es la gramática del verbo "poder". La única respuesta posible es una experiencia del lenguaje. ${ }^{14}$

Esta experiencia de la potencia de hablar no ha podido ser hecha en la infancia porque en ella se ha constituido. Sólo una vez que se alcanza la condición de hablante, se está en posesión plena de la capacidad de hablar y, por ende, de darle una nueva significación teórica y práctica a esta potencia. Es

${ }^{14}$ Ibidem, p. 219. 
a esta potencia de decir tanto como de callar, pero también al límite de lo que puede o no puede ser dicho, que remite la experiencia de la cultura en sus más diversos registros, desde la matemática hasta el arte, desde la política y la creación de las instituciones sociales y sus procesos de legitimación, hasta la crítica de las ideologías, la ética y la filosofía.

\section{E1 laboratorio de Wittgenstein}

\subsection{El experimentum linguae en el Tractatus}

Toda la obra de Wittgenstein, en especial después del Tractatus, puede leerse como un campo de experimentación para realizar la experiencia del lenguaje. Pero las bases ya estaban dadas en su primera obra. De la mano de Wittgenstein iremos de paseo por las amplias instalaciones de su laboratorio. Ahora bien, si no queremos perdernos en él, conviene saber lo que buscamos. Para ello, tengamos presente lo que asumiremos como una indicación o regla gramatical (RG) para el uso del concepto de experiencia, a saber:

RG: cuando tenemos experiencia de $x$, tenemos también experiencia de sus límites, en el sentido de que somos capaces de identificarla, y, por ende, de distinguirla de lo que no es $x$.

Al sostener RG, limitamos el alcance del concepto de experiencia a la vivencia consciente, sin rechazar sino sólo dejando de lado la idea de una experiencia inconsciente. La justificación para esta limitación está en que nos interesa la apropiación consciente y efectiva, por parte del hablante, de sus capacidades lingüísticas, no el estudio de estas capacidades como condición natural previa, ni como factor en una explicación causal o funcional de las capacidades implicadas.

Entonces, si la variable $x$ es interpretada como denotando al lenguaje, hacer o tener la experiencia del lenguaje implica la vivencia comprensiva de su límite, esto es, de su distinción del no lenguaje. Ahora sabemos qué buscar en el laboratorio de Wittgenstein: experimentos que nos permitan hacer esta experiencia, esto es, trazar la distinción entre lenguaje y no lenguaje o, como llegaremos a ver más claro, entre sentido y sinsentido, que es un objetivo estratégico de la filosofía del autor vienés. 
Si aceptamos ser guiados en nuestro periplo por el propio dueño de casa, conviene atender a lo que el autor de la obra enunció como su objetivo más profundo, cuando le indicó al editor Ludwig von Ficker que, para comprender el punto central del libro, que según le decía era ético, leyera el prefacio y la conclusión. De acuerdo a esta indicación, lo que Wittgenstein pretendió hacer en el Tractatus, fue mostrar que todo lo que puede decirse, puede decirse claramente, y que hay lo que no puede ser dicho, y que por ello hay que callar.

Ahora bien, para lograr establecer esto, se debía trazar el límite entre lo pensable y lo no pensable, pero, se nos dice, esto no puede hacerse porque no puede pensarse lo no pensable. Sin embargo, sí se puede trazar, en el lenguaje, el límite entre lo que puede decirse con sentido y lo que puede decirse sinsentido, asimilando el sinsentido al no pensamiento y el sentido al pensamiento. Debemos ahora preguntarnos si esta operación es clara o encierra, en su oscuridad, la clave oculta de la experiencia del lenguaje en el Tractatus.

Lo primero que resulta obvio es que en el prefacio se acepta una diferencia entre el límite entre pensamiento y no pensamiento, por un lado, y entre sentido y sinsentido por el otro, pues si no se reconociera esta diferencia, o bien ambos o bien ninguno sería trazable. Sin embargo, en segundo lugar, algo no debe ser diferente en el trazado de esos límites, para que uno de ellos represente al otro. En tercer lugar, si el límite es trazable en el lenguaje, entonces en él se puede hacer la experiencia de ese límite, -y ciertamente el Tractatus determina claramente las condiciones para hacerla-. Veamos cómo se concilian las dos primeras consecuencias entre sí y cómo se logra, además, la tercera.

No olvidemos que la distinción entre sentido y sinsentido será el instrumento para garantizar la distinción entre lo que puede y no puede decirse. Luego, ha de encontrarse un vínculo entre lo decible -el sentido-y lo indecible -tal que esto último se manifieste a través de lo primero, pero sin ser dicho, pues si lo fuera, necesariamente se produciría el sinsentido, índice de lo no pensable. Y bien, es de sobra conocido que lo que para Wittgenstein llegó a constituir el problema principal de la filosofía, es el recurso para lograr este peculiar anudamiento: la distinción entre decir y mostrar.

Si aplicamos RG al sentido, lo que resulta es que al hacer la experiencia del sentido, hacemos también la experiencia de su límite, esto es, de su 
diferencia con el sinsentido. Como sabemos, el sentido, lo propiamente decible y pensable, sólo puede ser alcanzado por un hecho de lenguaje: por la proposición. Y bien, toda proposición hace a la vez dos cosas cuando es verdadera: dice -asevera- que las cosas están así y asá, y muestra lo que la misma tiene en común con lo que la hace verdadera, su sentido ${ }^{15}$. Previamente a alcanzar esta articulación entre decir y mostrar, el Tractatus ha articulado otra tesis esencial: que cada proposición atraviesa el entero espacio lógico, la totalidad del lenguaje, con los solos recursos que la hacen capaz de ejercer su trabajo de decir y mostrar, esto es, el significado de sus partes. ${ }^{16}$

Resumamos esta trama en términos de nuestro experimento: cada hecho del lenguaje es una ocasión para hacer la experiencia del todo del lenguaje, y con ello, la experiencia de su límite, aquello que lo articula con el sinsentido. En consecuencia, este límite no puede decirse precisamente porque se muestra cuando se dice algo. De modo más directo, el lenguaje como un todo no puede ser dicho, pero se muestra cuando hay lenguaje, lo que ocurre en cada proposición -sea verdadera o falsa-.

Si retornamos a las tres consecuencias formuladas, advertimos que se establecen aquí dos relaciones para el pensar: una con lo que se dice y otra con lo que se muestra o se da a ver. El elemento de la mostración, al menos analógicamente, instala en el corazón de la semántica, una dimensión de sensibilidad que nos habilita a hablar de experiencia. Es gracias a la heterogeneidad radical entre decir y mostrar, -afirmada por si quedara alguna duda en TLP 4.1212: "Lo que se puede mostrar no puede decirse"17 -, que damos cuenta de la experiencia de la trascendentalidad del lenguaje en el lenguaje.

Por otra parte, dado que este es, según el Tractatus, el único modo legítimo de desarrollar la semántica, el modo en que esta estructura la experiencia a través del lenguaje no se refleja adecuadamente en la formulación teórica de la semántica de un lenguaje, sino haciéndola sensible en el reconocimiento de lo que se muestra, siendo ello mismo indecible (a diferencia

15 Tractatus Logico-Philosophicus (TLP), Madrid, Alianza, 1973, proposición 4.022, p. 75.

${ }^{16}$ TLP, proposición 3.42, p. 69.

${ }^{17}$ TLP, p. 87. 
de como aparece la semántica en las filosofías de Frege y Russell, inspiradoras de la del propio Wittgenstein).

Otra manera de comprender la naturaleza de la experiencia de los límites del lenguaje, que es la que hace posible la elucidación de la experiencia del lenguaje como tal, al menos en el experimento del Tractatus, es reparar en que los límites del lenguaje sólo cuentan desde dentro del lenguaje. Se trata evidentemente de un sentido anómalo o extendido del concepto de experiencia, pues pareciera que en toda experiencia, o al menos en la experiencia perceptiva, un límite puede verse tanto desde adentro como desde afuera, como por ejemplo el límite de una habitación.

Pero esto no es trasladable al lenguaje como un todo, excepto por el recurso al metalenguaje. Sin embargo, cuando con un metalenguaje nos referimos a un lenguaje-objeto, lo que en verdad hacemos no es capturar al lenguaje en su funcionamiento, pues esto queda ahora del lado del metalenguaje, mientras que el lenguaje-objeto no es ya usado sino mencionado.

En conclusión, como quedará más claro aún con los recursos de los que dispondrá Wittgenstein en la segunda etapa de su filosofía, al igual que en el Tractatus no se podía trazar la frontera entre lo pensable y lo no pensable, con lo que no se puede pensar ilógicamente, en relación al lenguaje no se puede trazar el límite entre el lenguaje y el no lenguaje, sino sólo exhibirlo dentro del lenguaje mismo. Pero ello no suprime el afuera del lenguaje, sino que, por el contrario, lo articula y vuelve significativo en el interior del mismo.

\subsection{E1 experimentum linguae en Investigaciones y otros textos}

Mucho se ha discutido si en la obra de Wittgenstein hay una continuidad o si es dable distinguir lo que se dio en llamar "primer, segundo, tercero, cuarto o último Wittgenstein". No es de nuestro interés sumar aquí elementos para decidir algo estratégico al respecto. Sin embargo, nos interesa destacar una línea de continuidad, más allá pero también a través de las diferencias. Esto sería tomarnos en serio, además, lo que Wittgenstein dijo en el prólogo a las Investigaciones filosóficas, en donde afirma que en algún momento le pareció adecuado publicar ese "álbum de paisajes o cuadros" junto con el Tractatus, 
porque creía que sólo a la luz de ese contraste entre su nuevo y su viejo modo de pensar podía comprenderse su trabajo, en ese entonces en curso.

Es obvio que las diferencias que se establecen en una comparación cobran su mayor importancia cuando se las evalúa sobre el fondo de aquellas semejanzas que hacen posible o al menos relevante la comparación misma. Y bien, lo que permanece como hilo que atraviesa y reúne las perlas del collar, es lo que Alberto Coffa señaló como el efecto más perturbador que el Tractatus ejerció sobre los miembros del Círculo de Viena: la adopción, como un principio activo de su concepción filosófica, de lo que llama "facticidad del significado". El reconocimiento de la facticidad del significado consiste, según Coffa, en el reconocimiento de que, antes de la especificación de cualquier lenguaje, ya debemos contar con las condiciones generales que establecen el límite entre sentido y sinsentido, entre la significatividad y la no-significatividad. Pero este límite es, si se me permite reiterar el oxímoron que he usado, un hecho trascendental. 18

En la sección anterior presentamos el experimento tractatiano que nos permitió explicitar esta facticidad del significado, pues hacer la experiencia del lenguaje es precisamente reconocer esa facticidad. Lo que ahora debemos hacer es encontrar algunos experimentos que en los textos posteriores de la obra de nuestro filósofo logran el mismo objetivo.

La tarea puede resultar controvertible, porque es bastante claro que algo notable varió entre el Tractatus y la obra posterior: el elemento convencional adopta en esta última un peso que no tenía en la primera, debido a que en aquélla la facticidad en cuestión se expresa en un esencialismo que Wittgenstein rechaza explícitamente en los textos escritos a partir de los años treinta.

No argumentaré a favor de la continuidad de la perspectiva de la facticidad del significado en forma directa, lo que he hecho ya en otro lugar. ${ }^{19}$

18 Coffa, Alberto, The Semantic Tradition from Kant to Carnap: To the Vienna Station. Cambridge, Cambridge University Press, 1993. Hay edición en español: La tradición semántica. De Kant a Carnap, (vol. I y II), México, UNAM, 2005. Ver especialmente capítulo 17.

${ }^{19}$ Cabanchik, Samuel M., "Facticidad del significado y exigencia comunitaria en la filosofía del último Wittgenstein", en Fernández Moreno, Luis (ed.), Para leer a Wittgenstein. Lenguaje y pensamiento, Madrid, Biblioteca Nueva, 2008. 
En todo caso, lo haré de modo indirecto, al explicitar la matriz común de los experimentum linguae a los que pasaremos revista, así como a algunos detalles de los mismos.

La matriz a la que me refiero resulta de la extensión de la familia de fenómenos del ver- cómo, del notar un aspecto, de la que Wittgenstein se ocupó especialmente en Investigaciones II, XI, ${ }^{20}$ como método general para la caracterización del método de la filosofía. ${ }^{21} \mathrm{La}(\mathrm{RG})$ se enuncia en este caso así:

RG1: a partir de tener la experiencia de $x$, podemos hacer la experiencia de verlo como $y$, con lo que hacemos también la experiencia de sus límites primero los de $x$, luego los de $y$ - y su mutua transformación.

El locus originario de esta experiencia es, paradigmáticamente, ciertas experiencias perceptuales de figuras, como la famosa de conejo-pato. Lo característico de este fenómeno es que se trata de ver una interpretación o un pensamiento, lo que también, usando una expresión del propio Wittgenstein, podemos describir como tener una vivencia del significado. Nuevamente aquí nos topamos con un anudamiento entre sensibilidad y significación, en el sentido de hacer de una característica semántica, el objeto de una experiencia.

${ }^{20}$ Wittgenstein, Ludwig, Investigaciones filosóficas, Barcelona, Altaya, 1999, pp. 445 a 523. ${ }^{21}$ Para un desarrollo amplio de esta extensión, consúltese Baker, Gordon, Wittgenstein's Method. Neglected Aspects, Oxford, Blackwell, 2004. En resumen, la estrategia de Baker es la siguiente: caracterizar la gramática del ver-cómo, luego extender su aplicación desde los casos del notar aspectos en la percepción de figuras, a la consideración de la diversificación de concepciones. En tercer lugar, interpreta que la representación perspicua sobre la gramática del lenguaje debe entenderse por analogía con el ver aspectos, precisamente como una diversificación en las concepciones de nuestra gramática. Entonces, las descripciones y juegos de lenguaje que se articulan en los textos de Wittgenstein serían vehículos del ver-cómo aplicados al lenguaje como tal. Esta interpretación global del método en Wittgenstein es convergente con la orientación que subyace al presente trabajo, pues podría decirse, ahora ya en mis términos, que el trabajo filosófico de Wittgenstein resulta ser, vía la técnica del símil en el sentido del ver-ómo, lo que llamamos experiencia del lenguaje, y su aporte principal a la cultura filosófica es justamente proporcionarle al lector un medio para que haga su propia experiencia del lenguaje y con el lenguaje. 
En el proceso de ver algo como algo diferente, lo que es objeto de sensación no cambia, como tampoco cambian cada una de las palabras y los conceptos que aplicamos a lo que vemos. Sin embargo, lo que se ha transformado como en un fulgor es el modo de verlo, fusionado ahora con un pensamiento o significación.

Pero RG1 sólo establece una posibilidad. Wittgenstein sostiene que, así como quien no deja de tener la experiencia perceptual primitiva podría no obstante ser incapaz de notar el cambio de aspecto, alguien podría, a los fines de la aplicación plena del lenguaje, disponer correctamente de todos los elementos sin ser capaz de tener la aludida vivencia del significado. En resumen, se puede hablar un lenguaje y sin embargo ser "ciego al significado", debido a que si bien se tendrían los significados primarios de las palabras y sus conceptos, faltaría la capacidad para la captación de los significados secundarios, esto es, para ver conexiones, establecer diferencias y semejanzas, en fin, para construir una distancia crítica en relación al código de la lengua y así modificar y variar las representaciones de nuestra gramática, algo esencial al ejercicio de la filosofía, pero quizá algo esencial a la condición misma de ser hablante de una lengua. En fin, en nuestros términos, diríamos que quien padeciera la ceguera para ver aspectos y tener la vivencia del significado, no sería capaz de hacer la experiencia del lenguaje.

Pero este "experimento" adquiere su condición crucial respecto de la experiencia del lenguaje, cuando nuestra RG1 varía en esta otra, anómala:

RG2: hacer la experiencia de $x \operatorname{como} x$, al verla como $-x^{22}$

\footnotetext{
${ }^{22}$ Massimo De Carolis, en Una lettura del "Tractatus" di Wittgenstein, Napoli, Cronopio, 1999, articula la profunda conexión entre el Tractatus y las Investigaciones, al interpretar los enunciados éticos que Wittgenstein formula en su famosa Conferencia sobre ética, y que deben entenderse como complementarios de su primera obra, a partir de la descripción del notar un aspecto de la que nos ocuparemos en la próxima sección y que recién se desarrolla en Investigaciones. Recordemos algunos de ellos: "me maravillo de la existencia del mundo y del lenguaje", "vivo la existencia del mundo y del lenguaje como un milagro”. En una lectura de notable perspicacia, De Carolis señala, por un lado, la peculiaridad modal que los mismos suponen, y, por otro lado, el hecho de que, en virtud de ello, se vuelven ejemplos extremos pero también paradigmáticos de los fenómenos del ver-cómo. En efecto, de acuerdo con el Tractatus, los hechos son
} 
Una aplicación de esta operación parece hacer Wittgenstein en el siguiente texto, en el que $x$ se interpreta como realidad, -pero que también podría ser entendido como sentido-:

La sensación de la irrealidad del entorno. En alguna ocasión tuve esa sensación y muchos la tienen antes del inicio de una enfermedad mental. Todo parece de alguna manera irreal; pero no como si uno no viera las cosas clara o borrosamente; todo tiene su aspecto usual. ¿Y cómo sé que otra persona ha sentido lo mismo que yo he sentido? Porque usa las mismas palabras que yo mismo encuentro apropiadas. Pero ¿por qué elijo la palabra "irrealidad" para expresarlo? (...) La elijo debido a su significado. Pero seguramente no he aprendido a usar esta palabra en su significado de una sensación. No, pero la he aprendido con un significado particular y ahora lo uso espontáneamente de esa manera. Se podría decir...cuando he aprendido esa palabra en su significado normal, elijo ese significado como símil para mi sensación. Pero, naturalmente, no se trata aquí de un símil, de una comparación de la sensación con otra $\operatorname{cosa}^{23}$.

contingentes, tanto como las proposiciones que los figuran, pero ¿cómo afirmar lo mismo del mundo y del lenguaje en los que esos hechos y proposiciones se dan? Es como si Wittgenstein tratase aquí al mundo mismo como un hecho del mundo, lo que es un sinsentido en el concepto técnico de su temprana obra o, también podríamos decir, como si tratase la determinación ser el lenguaje, como un argumento de sí misma en tanto función proposicional. Es claro que de los simples (objetos y nombres) no cabe decir que son contingentes como sus combinaciones efectivas. Esta distorsión modal logra ser expresada, como un caso extremo del ver algo como algo. Para ver cómo se aplica a estas enunciaciones nuestra regla, reformulémosla ligeramente a partir de nuestro esquema: "hago la experiencia de $x$ (el mundo, el lenguaje) como tales, al verlos como pudiendo no ser el mundo o no baber del lenguaje". Una de las características de los fenómenos del ver-cómo, es que en ellos cambia el modo de ver, pero no lo visto. De la misma manera, no hay ningún hecho que se cambie o altere si vemos su conjunto como pudiendo no haber sido, de la misma manera que nada suma o quita al lenguaje, imaginar que podría no haber habido lenguaje alguno. Aquí, como en el experimentum linguae que veremos más adelante a propósito del contraste entre realidad e irrealidad, no hay un símil auténtico. Por ello, se trata de un caso de ver $x$ como $x$, no de ver $x$ como $y$. El recurso para lograr que parezca que hay un símil donde no lo hay, es pasar por la comparación de $x$ con $-x$.

${ }^{23}$ Wittgenstein, Ludwig, Observaciones sobre la filosofía de la psicología, México, UNAM, 1997, vol. I, sección 125, p. 26 e. 
Considero este pasaje iluminador para extender las experiencias de vercómo a efectos de hacer un nuevo experimentum linguae. Considerémoslo en detalle.

El contraste establecido entre real/irreal engloba tanto el hecho de que a ambos lados de la barra se tiene el mismo mundo percibido como el mismo lenguaje. Sin embargo, hay una vivencia que me permite expresar este contraste, pero que no se apoya en un símil efectivo, una auténtica comparación, sino en una que se realiza, por así decir, más allá de los hechos, los perceptuales y los del lenguaje.

En lugar de hablar del par real/irreal, sería perfectamente legítimo hablar del par sentido/sinsentido, con lo cual estaríamos en presencia de un experimento en el que se muestra la vivencia correspondiente a hacer la experiencia del lenguaje como tal. Yendo más lejos aún, lo que el símil anómalo nos permite considerar, es que la experiencia de lo real que hiciera un hablante ciego para el significado tendría el aspecto de lo irreal.

Esto agrega una complejidad importante a nuestro "modelo". En efecto, es como si a causa de la falta del ejercicio de la vivencia del significado aquí implicada, el hablante, al no poder hacer la experiencia del límite del lenguaje, no pudiera hacer la experiencia plena del sentido, por no poder trazar el límite entre sentido y sinsentido desde dentro del lenguaje. Inversamente, sería como si viera el lenguaje desde fuera, sin participar de él. En otras palabras, como si no hubiera accedido aún al sentido y tuviera que, por así decir, obtenerlo directamente de un aparato lingüístico sin ser un hablante del mismo, -y de ningún otro, claro-.

Otro "experimento", uno de esos que solemos llamar "experimentos mentales" en filosofía, parece dirigido por Wittgenstein a ilustrar la diferencia entre concebir al lenguaje como un mecanismo y concebirlo como lo hacemos naturalmente, como hablantes del lenguaje. Se desarrolla en una serie de largas y algo barrocas secciones de la Gramática filosófica. ${ }^{24}$ Es imposible citar aquí enteramente esas secciones, por lo que ofreceré una lectura de los mismos en

\footnotetext{
24 Ver las secciones 135 a 142 en Wittgenstein, Ludwig, Gramática filosófica, México, UNAM, 1992, pp. 367 a 385. Consúltese también Investigaciones filosóficas, ed. cit., p. 331, sección 495.
} 
la que intentaré resumir lo esencial de la argumentación a los fines de nuestra presente indagación.

Wittgenstein nos invita a concebir un lenguaje como el descubrimiento o la invención de una máquina, cuyo funcionamiento correlaciona los estímulos -órdenes e instrucciones ejecutadas en un teclado- con las respuestas conductuales previstas por las órdenes y por el funcionamiento del mecanismo. (En dicha suposición, podemos restringir esta "máquina de lenguaje" a una lengua conocida, como el alemán o el español).

En este experimento, la regla gramatical vuelve a ser RG1, ya que se trata de ver a $x$ como $y$, donde " $x$ " es lenguaje e " $y$ " es la máquina, es decir que aquí sí hay una comparación efectiva. Un símil semejante, nos dice Wittgenstein, dejaría afuera el fenómeno del lenguaje, aunque preservaría la descripción en términos de estímulo-respuesta, en donde la idea de un funcionamiento correcto de la máquina se identificaría con la obtención de los resultados previstos o deseados. Ahora bien, querríamos decir por nuestra parte, ¿no es esta una comparación entre hacer la experiencia del lenguaje desde su interior y hacerla desde su exterior?

Wittgenstein no establece explícitamente el contraste interior/exterior sino otros que interpreto como semejantes. Uno de ellos es que la máquina de lenguaje, su gramática, se determina en la forma de conexiones causales, lo que difiere de nuestro concepto natural de lenguaje. Sin embargo, visto desde fuera como un mecanismo, en nada diferiría de lo que llamamos comunicación, y podría resultar circular aducir que la diferencia está en que en el concepto natural de lenguaje se incluye la representación de seres vivientes que aplican signos, pues a la hora de caracterizar a los hablantes como vivientes, reintroduciríamos, probablemente, la capacidad de usar signos en un lenguaje como su rasgo característico, que es lo que queríamos obtener con nuestra descripción.

Si el contraste entre nuestro concepto natural de lenguaje y el que se describe como una máquina de lenguaje no puede establecerse a través de los conceptos de comunicación, vida o el par desde dentro/desde fuera, ¿qué podría establecerlo? Según nuestra lectura, para ese fin Wittgenstein varía el experimento comparando al lenguaje como una actividad que se practica y en la que se combinan los signos en proposiciones y frases, con operaciones con 
señales independientes entre sí, que lograrían los mismos efectos que la práctica natural. Su respuesta es, nuevamente, que la diferencia está en lo que vuelve a llamar fenómeno del lenguaje, descripto como un cálculo o como una práctica en la que introducimos convenciones y aplicamos reglas.

¿Cuán lejos nos permite llegar el símil? Pareciera indicar que el concepto de lenguaje que utilizamos para describir y comprender nuestro uso natural de las palabras no puede reducirse a una conexión mecánica cuya autorregulación se determinaría como lo hace una conexión causal, en la que se busca obtener ciertas respuestas a partir de ciertos estímulos. Si describiéramos nuestro lenguaje según esta concepción, lo haríamos como alguien que no participa en él, sino que lo trata igual que a cualquier regularidad empírica que observa, o con la que interactúa como con un instrumento del que se vale para ejecutar una aplicación meramente causal.

A través de este símil, lo que apreciamos es que la experiencia del lenguaje, que en este caso podemos interpretar que se expresa en el contraste entre una descripción no participativa del mismo contra otra participativa, no puede hacerse sin que para nosotros el lenguaje sea un fenómeno, esto es, algo de lo que tenemos una vivencia, por así decir. Pero esta vivencia no se apoya en un proceso o episodio psicológico, sino en la descripción y comprensión misma del fenómeno como una práctica dada en la que nos encontramos. Es decir, la vivencia no explica al lenguaje, sino que se da conjuntamente con él y en medio de él.

El símil de la máquina es usado reiteradamente por Wittgenstein en las Investigaciones: en el caso de la máquina de leer, ${ }^{25}$ en el de la máquina como símbolo ideal en el que todos sus movimientos - los de la máquina- están ya contenidos, presentes y determinados en el mecanismo, ${ }^{26}$ o para señalar el fracaso de representar la idea de uso correcto de un signo para nombrar una sensación,

25 Investigaciones filosóficas, pp. 159 y 175, secciones 157 y 170, respectivamente

26 Investigaciones filosóficas, pp. 193 a 197, secciones 193 y 194. Para un esclarecedor análisis de estos textos, su implicancia para comprender la concepción del lenguaje contra la que dirige su crítica la propia concepción wittgensteiniana y su vínculo con el problema del seguir una regla, véase Gómez López, César, Significado y libertad. Un ensayo en filosofía del lenguaje, Madrid, Siglo XXI, 2003. 
como si fuera una regularidad aislada dentro de una máquina, ${ }^{27} \mathrm{o}$ para explicitar algún rasgo de la gramática de la palabra "pensar" 28 . El elemento común en estas comparaciones es el contraste entre la representación del funcionamiento del lenguaje como un mecanismo físico, psíquico o idealizado, y la descripción de una práctica participativa en términos de juegos de lenguaje y formas de vida.

A través de este esfuerzo por ver el uso del lenguaje como un mecanismo cuasi-causal, podemos concebir el sentido que podemos darle a la expresión "hacer la experiencia del lenguaje". Podemos decir que sólo quienes participan del lenguaje pueden hacer su experiencia, y que no logramos dar cuenta de la misma si lo pensamos como un mecanismo a través de imágenes asociadas al funcionamiento de una máquina. (Sin embargo, tal experiencia no es inefable sino enteramente describible, al describir al lenguaje como una práctica global y colectiva articulada en formas de vida. Por el contrario, lo que nos induciría a representarnos dicha experiencia como inefable, es un efecto de verla como una clase de mecanismo).

La descripción de la desemejanza entre el lenguaje como un mecanismo y el lenguaje como una práctica, es una aplicación muy peculiar del notar un aspecto. En efecto, en esta comparación se nos invita a ver nuestra condición de hablar un lenguaje como un dispositivo que, por un lado, fingiría o simularía sus rasgos exteriores, convirtiendo, por otro lado, sus concomitantes vivenciales en fantasmas que habitan en los intersticios de sus engranajes y que, además de volverse sombras incomprensibles aún en el propio caso, estarían demás, no ejercerían trabajo alguno.

Veamos más de cerca cómo a través de estas aplicaciones del fenómeno del ver-cómo, logramos hacer nuestro experimentum linguae. Observemos que en la primera situación en la que uno de los "sujetos" de experiencia percibe los hechos con la vivencia de su realidad mientras el otro los percibe a través de una neblina de irrealidad, es en la práctica lingüística común en donde, quien no padece "ceguera para lo real", por así decir, logra ver lo real como real por su diferencia con lo irreal. En cambio, el otro sujeto, envuelto en su irrealidad, funciona en esa comunicación y en su relación con el mundo percibido y

${ }^{27}$ Investigaciones filosóficas, p. 233, secciones 270 y 271.

${ }^{28}$ Investigaciones filosóficas, pp. 275 y sig., secciones 359 y 360. 
concebido, como lo hace la "máquina de lenguaje" en el otro símil. La máquina o el afectado en su experiencia, logran comunicarse con el hablante a pesar de que sólo para éste se establece el contraste entre, por un lado, participar plenamente del fenómeno del lenguaje y de su adecuación con la experiencia de lo real, y por el otro, intercambiar señales de un código o meramente adaptarse a regularidades empíricas.

Variemos ahora nuestro experimento, adscribiendo la vivencia de estos contrastes a un mismo hablante. Lo que este alcanza con estas prácticas de vercómo, es la experiencia de los límites del sentido, toda vez que, manteniéndose en el lenguaje, lo concibe representándoselo como un mecanismo cuasi-causal, en una palabra, no como lenguaje sino como su simulacro (aquí extendemos el concepto de lenguaje para abarcar, también, la experiencia del sentido de lo real en su conjunto).

La idea de hacer una experiencia de la trascendentalidad del lenguaje, viene dada aquí por el hecho de que es sólo desde la perspectiva del hablante que el lenguaje aparece como condición del conocimiento del mundo y de la comunicación auténtica. La máquina o el alienado no participan del lenguaje, sino que interactúan con los efectos de su práctica y, en consecuencia, no tienen la articulación natural y orgánica entre las palabras y las cosas. Es en este punto que hacer la experiencia del lenguaje nos habilita a la vez, a hacer la del no lenguaje, es decir, a estar "en los dos lados del lenguaje". Si no fuéramos capaces de esto, ¿no quedaríamos reducidos a la situación de la máquina o del alienado?

Es como si el conocimiento filosófico lograra regresar a esa instancia en la que debimos estar, en nuestra infancia, al transformarnos de vivientes no hablantes en hablantes, es decir, al pasar desde el lado del no lenguaje al del lenguaje. Pero en ese entonces, dado que aún no estábamos en el lenguaje, no podíamos hacer la experiencia de sus límites. Por el contrario, el experimentum linguae nos hace pasar del lenguaje al no lenguaje, permitiéndonos apropiarnos de nuestra potencia lingüística en su pureza, pues en ella encontramos anidada también, la impotencia en la que se sumiría aquel que perdiera el lenguaje y, de ese modo, se volviera in-fante otra vez.

¿No es el caso de la filosofía? Escribió Wittgenstein: 
Con frecuencia, los filósofos son como niños pequeños que empiezan por hacer rayas caprichosas con su lápiz sobre un papel y después preguntan a los adultos: ‘qqué es?'. Lo que sucedió fue esto: el adulto le había dibujado con frecuencia algo al niño y le había dicho: 'esto es un hombre', 'esto es una casa', etc. Y ahora el niño pinta también rayas y pregunta: '¿qué es esto? 29

Dijimos "estar a los dos lados del lenguaje". Es el viaje de la filosofía cuando se dispone a hacer el experimentum linguae. Exploremos ese viaje de la mano de la literatura, como si fuera un nuevo experimento de estilo wittgensteiniano. En un célebre pasaje de Alicia a través del espejo de Lewis Carroll, se desarrolla un diálogo entre Alicia y Humpty Dumpty al otro lado del espejo. Por vía de una frecuente analogía filosófica entre el lenguaje y el espejo, introduzco la hipótesis de que en dicho diálogo se manifiesta un lenguaje "del otro lado del lenguaje” por así decir. ¿Qué aspecto del lenguaje se nos muestra allí?

Podríamos servirnos del diálogo entero para ilustrar el funcionamiento del lenguaje desde la perspectiva de la polaridad normal/normativo. En un primer momento, Alicia se dirige a Humpty Dumpty haciendo un uso normal del lenguaje, tal como se deja ver por el contraste con los comentarios, preguntas y respuestas de Humpty Dumpty. También se dice a sí misma que su interlocutor parece jugar con el lenguaje en vez de realmente conversar, etcétera. Avanzada la escena, es ella misma la que comienza a asumir el supuesto diálogo como un juego y, jugándolo, comienza a intentar descolocar a Humpty Dumpty como antes éste lo hacía con ella.

El intercambio verbal se aproxima más y más a un combate hasta llegar al clímax, lo que ocurre cuando Alicia pretende recuperar el control del diálogo sometiendo a Humpty Dumpty a la literalidad de una definición de significado, sin admitir el uso alusivo o metafórico que éste había hecho de la palabra "gloria". En ese punto el diálogo discurre en estos términos:

${ }^{29}$ Wittgenstein, Ludwig, Observaciones, Siglo XXI, México, 1981, p. 39. 
Cuando yo empleo una palabra -dijo Humpty Dumpty con el mismo tono despectivo-, esa palabra significa exactamente lo que yo quiero que signifique, ni más ni menos.

-La cuestión es saber -dijo Alicia- si se puede hacer que las palabras signifiquen cosas diferentes.

-La cuestión es saber -dijo Humpty Dumpty- quién dará la norma... y punto. ${ }^{30}$

Si nos asomamos al otro lado del espejo/lenguaje, tal como se lo escenifica en la obra de Lewis Carroll, comprendemos que lo que perdió Alicia al atravesarlo es la forma de vida. El modo en que se desarrollan los diálogos de Alicia con sus diferentes interlocutores, Humpty Dumpty incluido, evidencia que los juegos de lenguaje que no se ajustan a normatividad inmanente alguna, no arraigan en ninguna forma de vida.

Alicia tiene razón en advertirle a Humpty Dumpty que tal vez no pueda hacerle decir al lenguaje lo que se le ocurra, pero éste muestra en su exceso, en su excepcionalidad, que el lenguaje no tiene bases más seguras que el acuerdo dado en las formas de vida comunitariamente compartidas. Pero, sobre todo, Humpty Dumpty es un personaje estratégico, porque expresa la potencia normativa del hablante. Si éste no la retuviera, la normalidad que lo constituye como tal lo sumiría en la impotencia de un lenguaje sin vida.

Desde esta perspectiva, el espejo se ha roto en sus pretensiones representacionales, pero con todo, es lo único que tenemos para protegernos de la deriva patológica por la que somos arrastrados cuando, en tanto hablantes de un lenguaje, queremos instaurar nuestra soberanía más allá de él mismo: sin espejo sólo nos quedaría el delirio en el que toda referencia y todo sentido se perderían, pero sin fractura no avizoraríamos que la potencia normativa se encuentra en nosotros, esa que nos mantiene abiertos a la renovación comunitaria de nuestras formas de vida.

La moraleja del "diálogo" es clara: hay una norma del significado inmanente al uso del lenguaje, que aspira a dar estabilidad y normalidad a este,

${ }^{30}$ Carroll, Lewis, Alicia en el país de las maravillas. A través del espejo. La caza del Snark, Barcelona, Editorial Óptima, 2000, pp. 185 y sig. 
pero que deja abierta la cuestión acerca de cuál es la fuente normativa de la que proviene, y por qué dicha normatividad permanece abierta a la insuficiencia de su fundamento y a su siempre revocable estabilidad.

\section{Eticidad y politicidad del lenguaje}

La filosofía de Wittgenstein reconoce en una misma condición tanto el origen de la carencia de fundamento como de la creatividad normativa en el uso del lenguaje. Esta condición puede describirse en los siguientes términos: la eficacia causal del ánthropos es función de una normatividad que, para ser operativa, requiere de nuestra propia capacidad para cumplir o cancelar la norma. Si se retiene el peso estratégico del lenguaje, la razón es que es en el lenguaje, justamente, donde esta capacidad alcanza su concreción básica y esencial. Remontando el camino filogenético, diremos que si la especie humana sobrevivió y se desarrolló, fue porque aquellos individuos ancestrales se acomunaron y se comunicaron; en el contexto ontogenético, por otra parte, se verifica que si el infante sobrevive y alcanza el status de lo humano, es porque logra soldarse en él su pobre eficacia causal natural, con la fuerza de la regla, fulcro de la potencia lingüística.

Pero si nuestra potencia causal queda supeditada a nuestra propia normatividad, ¿cómo podríamos discriminar entre lo normal y lo patológico, sea en la regla, sea en su aplicación? Se reconocerá en este tópico el legado wittgensteiniano, en ciernes en el Tractatus; plenamente desarrollado en las Investigaciones.

Reconocemos el nudo de esta problemática cuando reparamos en que, para "el animal dotado de logos", todo y nada puede ser igualmente significativo; que antes de la norma, ruido y señal se confunden. Ahora bien, no habrá ni ambiente ni mundo donde esta discriminación no sea operativa. Pero, precisamente, puesto que para el humano tal discriminación depende de su potencia normativa, igualmente capaz de producir el caso normal tanto como el patológico, su universo de sentido no se cierra sin abrirse; no se estabiliza sin exponerse a la amenaza de su cancelación repentina; no triunfa en el establecimiento y mantenimiento del sentido, sino admitiendo una y otra vez la perenne fragilidad de la norma, cuya certeza es concomitante con su 
propensión a fallar, lo que posibilita a su vez la creatividad más favorable, y la perversión más aberrante.

Frente a este panorama, se vuelve inexorable interrogar, con inclaudicable insistencia, cómo deben ser nuestras prácticas políticas, cuáles nuestras instituciones, tales que en su dinámica real consagren esta ambivalencia paradójica de lo humano, regulando el lazo social a través de la distinción entre lo normal y lo patológico, lo legítimo y lo ilegítimo, lo elogiable y lo condenable.

Y bien, este "estado de abierto" no es cancelable sin renunciar a la dimensión práctica de nuestro ser lingüístico. Dicho de otra manera, si hay ética y política, es a través de la eticidad y la politicidad del lenguaje mismo, que alcanza su plena dimensión pública en la experiencia del lenguaje celebrada en nuestro ser-en-común.

Ahora bien, si tanto la ética como la política son una condición de estructura, sin embargo, es propio de dicha condición poder ser realizada o no en nuestro ser social; que lo sea comporta una decisión, una actitud: ¿cuáles son las condiciones de esta actitud?

Volvamos a la ontogénesis del hablante. Lo que se produce en dicha génesis es la separación del ser vivo respecto de esa vida previa a la adquisición del lenguaje. Propondré entender esa separación en el modo del como no. Veamos en qué consiste dicho modo.

En un libro dedicado a interpretar en qué sentido el mesianismo se encuentra en San Pablo, Agamben ${ }^{31}$ explica el hos me -que traducimos por "como no"-, resultado de la vocación mesiánica, como revocación de toda vocación, en el sentido en que cada condición fáctica se pone en relación consigo misma para "ser revocada, puesta en cuestión sin ser alterada en su forma". Esto quiere decir que llorar como no llorando o reír como no riendo no es hacer otra cosa que llorar y reír, pero haciéndolo afectado por haber respondido a la vocación o llamado mesiánico.

${ }^{31}$ Agamben, Giorgio, El tiempo que resta. Comentario a la carta a los romanos, Madrid, Editorial Trotta, 2006. 
Avanzando en su comentario, Agamben destaca otra significación del como no: cada condición fáctica afectada por esta modalidad establece entre el agente/paciente de dicha condición y esta misma la relación de uso. En sentido global, según mi entendimiento de la lectura de Agamben, podemos decir que la vocación mesiánica afecta a la vida misma, al punto de que el ser viviente afectado por dicha vocación hace uso de ella en cada situación y cada condición, pero en cierto sentido no la posee permanentemente o, mejor dicho, no se identifica con ella.

Me permito ahora extender esta caracterización de las consecuencias del llamado mesiánico a la caracterización del modo en que es afectado el viviente cuando es llamado al lenguaje. Afectado por el llamado al lenguaje, el viviente sigue viviendo esa vida que vivía antes, pero en algún sentido lo hace como no viviéndola, al menos en el sentido previo en que era uno con su ambiente.

Vivir la vida como no viviéndola es, para el hablante, estar separado de su vida en la forma de comportarse consigo mismo y con el mundo que habita. Esta separación no es sin embargo aislamiento entre ambas dimensiones. Debe entendérsela con la lógica del como no: el ser hablante vive como no siendo el ser viviente que fue y, por ello y a pesar de ello, sigue siendo.

Dada la condición descripta: ¿qué relación guarda esta condición con el valor? ¿Es una condición necesaria, suficiente $-\mathrm{O}$ ambas cosas- para que haya valor? Esta no es una pregunta sencilla y llevaría mucha argumentación concluir algo preciso. Pero no necesitamos hacer eso sino algo más específico: responder si la condición viviente-hablante es necesaria o suficiente -o ambas cosas- para hacer de dicha condición un sujeto/objeto de valoración. En forma más directa: ¿podría una máquina artificial o un animal valorarse? ¿Podría un viviente-hablante no hacerlo? Mi hipótesis es que la normatividad específica del animal lingüístico lleva a éste a adoptar necesariamente una actitud frente a su condición y, por lo tanto, a valorarla, aun cuando dicha valoración pudiera no constituirse en juicio explícito y, más allá de que sea concebible que un artefacto o un animal sean capaces de valoración, esas valoraciones no provendrían de escisión alguna.

Tanto en la máquina como en el animal, tales improbables valoraciones constituirían estados positivos, resultantes a su vez de la aplicación de una norma inmanente a sus respectivas constituciones. Por el contrario, la 
separación que constituye al existente afecta a éste en su condición misma de un modo irreparable.

Una vez que la vida entra en el régimen del valor, admite las polarizaciones y conflictos propios de los valores. En consecuencia, la vida podrá adquirir para el existente un signo positivo o un signo negativo. $\mathrm{Y}$ bien, cuando la vida se carga con una valoración positiva, estamos frente a lo que llamaré gracia o felicidad, mientras que una vida desgraciada o infeliz es una vida negativamente valorada.

Es preciso distinguir aquí entre una noción adecuada y una inadecuada de felicidad. Una concepción adecuada de la felicidad tiene que dar cuenta de la separación constitutiva del viviente-hablante. Esto significa que para este tener una vida feliz será vivirla según la condición del como no antes referida. En cambio, cuando se representa la felicidad como un estado en el que el vivientehablante conquista una plenitud animal o angélica, sencillamente se aplica mal el concepto, pues ni los animales ni los ángeles pueden ser felices porque "feliz" se predica de aquellos seres que tienen una vida ${ }^{32} \mathrm{y}$, por ende, son capaces de valorarla positiva o negativamente, algo que, según nuestra conjetura inicial, está reservado a los vivientes-hablantes.

Apliquemos ahora este desarrollo a la perspectiva ética del "primer Wittgenstein". Como se recordará, Wittgenstein afirma que ningún hecho del mundo es en sí mismo valioso, sino que el valor es una propiedad del mundo o vida tomados como un todo. La vida feliz consiste en la adecuación de la voluntad con la vida; la desgraciada con su inadecuación. ${ }^{33}$ Esta diferencia se deriva, según la anotación del 29.7.16 del Diario filosófico, ${ }^{34}$ del modo en que se

${ }^{32} \mathrm{El}$ contraste se establece aquí entre "ser una vida y "tener una vida", con el sentido de señalar que el humano, en tanto animal que habla, es una vida sólo en el modo de tenerla, que implica su separación con la vida animal que hace uno con su ambiente. En cambio, del animal no hablante puede decirse que es una vida, y que ese es su único modo de tenerla, esto es, sin distancia con su condición de ser uno con el ambiente que habita. Podría también decirse esto: "el animal no hablante tiene ambiente y es una vida; el animal hablante tiene una vida y no tiene ambiente, al no ser uno con él".

33 En TLP 6.41, p. 197 de la edición citada.

34 Wittgenstein, Ludwig, Diario filosófico (1914-1916), Barcelona, Ariel, 1982, pp. 132 y sig. 
ejerce esa voluntad o deseo. El modo en que desea quien vive feliz es el de quien vive en el presente como no viviendo en el tiempo. ${ }^{35}$

Ahora bien, ¿qué otro sentido puede darse a este "vivir en el presente como no viviendo en el tiempo", esto es, como no siendo el presente viviente que sin embargo es, sino como la afirmación o aceptación de la vida del vivientehablante, que Wittgenstein distingue de la vida fisiológica, ${ }^{36}$ en tanto afectada por la escisión que la constituye? He ahí la concepción de la vida felizpropuesta por Wittgenstein: es la vida que se acepta a sí misma en su separación, pues sólo una vida así puede ser una voluntad, condición para que baya valor en la vida o mundo.

El panorama que se nos presenta ahora es el siguiente: ser feliz en el mundo, si se entiende como la aceptación misma de la condición humana en tanto viviente-hablante, no sólo es posible, sino que constituiría la única manera de asumir dicha condición adecuadamente. Vive una vida feliz quien se instala plenamente en la separación entre lenguaje y vida, gracias a la cual tiene una vida, a sabiendas de que por eso mismo no es una vida. Sólo los seres humanos podemos ser felices en este sentido.

En pocas palabras, cabe afirmar que la felicidad es la norma misma del animal que habla, por lo que no sólo podríamos, sino que deberíamos ser felices. De ahí que Wittgenstein escribiera el mandato: “isé feliz!”. Pero el cumplimiento de este deber no consistiría en ninguna forma específica de vivir la vida, excepto la de afectarse a sí mismo con la escisión constitutiva de una vida humana.

A través de dicha afectación, el modo en que ejercemos nuestra voluntad o nuestro deseo modifica a la vida de cada quien como una totalidad. Se introduce así en la vida un valor absoluto, que no sirve a los fines de indicar ningún curso de acción específico, para lo cual están los valores relativos dentro del mundo, pero que otorga a la vida como tal la fuente misma de todo impulso en dirección de cualquier valor o cualquier finalidad.

\footnotetext{
35 Ver en el Diario filosófico (1914-1916) la anotación del 8.7.16, donde Wittgenstein afirma: "Solo quien no vive en el tiempo, haciéndolo en el presente, es feliz", p. 129. 36 Diario filosófico (1914-1916), anotación del 24.7.16, p. 132.
} 
La vida sumida en los hechos o en el ser es una vida sin valor, pues para que haya valor han de rebasarse los hechos hacia lo que por definición no puede adquirir objetivación alguna, -lo que, si existiera, se impondría con necesidad imperativa al viviente. Por ello, porque en el mundo no hay necesidad, es que tampoco hay valor-.

La relación establecida entre vida y valor hasta aquí presentada se adecua a un principio de inmanencia, que en este contexto significa que la vida en tanto existencia separada, según el régimen del como no, absorbe enteramente el valor, impidiendo su alienación como objeto trascendente, es decir, su transformación en pseudo-hecho.

En este sentido, ¿por qué no concebir entonces una vida valorada como una vida transformada por una significación que la abarca como un todo, independientemente de los hechos que la componen, es decir, de cómo efectivamente sea el mundo? Es con este status que ha de concebirse la polaridad feliz-desgraciada respecto de la vida: no son cualidades de hecho, sino imaginarias, como las cualidades artísticas, dependientes de significaciones, de discursos.

El modo en que le es dado al yo ético o de la voluntad acceder a su mundo o vida como a un todo, nos indica que debemos concluir que solo imaginariamente es posible vivir la vida como un todo. Lo que nos permite entrever por qué Wittgenstein no realizó una distinción entre ética y estética, incluso entre ética y religión, por tratarse, como ya hemos dicho, de una actitud ante la propia vida vivida.

La construcción de esta totalidad exige la instancia imaginaria de una mirada contemplativa que sólo se sostiene imaginariamente. Pero si esta construcción depende de una mirada, que es un objeto imaginario, ¿no será también ese mundo del cual predico felicidad o infelicidad también imaginario? Mejor dicho, ¿no es la polaridad felicidad/infelicidad en cuestión, también ella imaginaria? ¿Y no lo sería de la misma forma cualquier valor?

Las respuestas no pueden ser sino positivas, pues como hemos ya indicado, un valor es un pseudo hecho, no pertenece a lo que hay, es más bien una hipóstasis. Su régimen es el de lo imaginario, como lo son los valores tales como la belleza, el bien, etc. 
Esta construcción imaginaria de la vida buena es solidaria con el modo en que Wittgenstein abrazó la facticidad del lenguaje en el Tractatus. Dado que la expresión de la ética no satisface las condiciones de sentido fijadas en dicha obra, sólo podía sobrevenir en el silencio místico. La filosofía se afirma como una ética aceptando decir el sinsentido, para que el factum del lenguaje encuentre su enunciación. El silencio se hace audible exclusivamente en primera persona del singular, solidariamente a la ecuación solipsismo $=$ realismo. ${ }^{37}$

Si bien Wittgenstein pretende que este solipsismo realista se muestre sin decirse, se ve llevado a decirlo, inexorablemente, en los sinsentidos filosóficos del Tractatus. He aquí una primera transgresión en la que la filosofía aparece aceptando el sinsentido para que un indecible finalmente se haga decible.

A esta transgresión la Conferencia sobre ética agrega otra: la enunciación del sinsentido ético ya ejemplificado en su expresión paradigmática: "vivo la existencia del lenguaje como un milagro". ${ }^{38}$ Ambas expresiones juntas, la del solipsismo lógico y la de lo que llamamos "solipsismo ético", conforman las dos caras con las que se presenta la afirmación del lenguaje como un factum originario e irreductible en el primer período del pensamiento de Wittgenstein.

Como dijimos, esta conceptualización de la ética ubica al valor como una envoltura imaginaria del mundo más allá del mundo, del lenguaje más allá del lenguaje. A continuación, intentaré mostrar que el nuevo modo de concebir la facticidad del lenguaje en el resto de su obra, permitirá a Wittgenstein trascender dicha construcción imaginaria habilitando, frente al solipsismo ético explicitado, un ethos comunitario, -no exento, por su parte, de estructuración imaginaria-.

En este punto no tenemos más que señalar que lo esencial de la perspectiva del lenguaje en "el último Wittgenstein", muestra que no hay primero hablantes qua individuos que luego se encuentran entre sí para interpretarse mutuamente, puesto que el individuo es ya comunitario, dado que

${ }^{37}$ Esta ecuación es propuesta por Wittgenstein en las sentencias 5.6 a 5.62 del TLP. Ver p. 163 de la edición citada.

${ }^{38}$ Incluida en Wittgenstein, Ludwig, Ocasiones filosóficas (1912-1951), Madrid, Cátedra, 1997. 
la comunidad es aquel plano, el lingüístico, que hace de un individuo un hablante. ${ }^{39}$

Debido a ese hiato, lo mismo que hace posible el ser-en-común del lenguaje, hace también posible el no ser-en-común de los hablantes; la facultad de entenderse es la imposibilidad de garantizar dicho entendimiento en algo estable y permanente, al abrigo de todo malentendido.

Ahora bien, es en ese hiato que debemos ubicar el ethos comunitario, como exigencia y como posibilidad. En virtud entonces del giro práctico que lleva a un nuevo modo de asumir el lenguaje como un factum, el "isé feliz!" de Wittgenstein alcanza una nueva dimensión. La exigencia de felicidad ya no se deja comprender en la perspectiva del solipsismo ético; ahora requiere de una realización comunitaria. ¿Pero en qué podría consistir dicha realización?

No se trata de que sea feliz la comunidad, sino de encontrar en la vida en común del lenguaje y de las formas de vida, la posibilidad misma de la vida buena. El primer paso es reconocer que no hay vida - ni buena ni mala- en el exilio de la vida común -que es la vida en común-. Es en medio de la vida en común que nos es dado todo: nuestra soledad tanto como la oportunidad de compartirla, la materia misma de nuestras emociones, deseos y elecciones, como también el orden moral que se edifica sobre ello: lo bueno y lo malo, lo justo y lo injusto; en fin, el cielo y el infierno de lo humano.

Desde este punto de vista, si bien Wittgenstein había movilizado ya en época del Tractatus los recursos suficientes para hacer de la filosofía misma una ética, aún estaba comprometido con ese atajo prematuro que queda inmejorablemente expresado en el solipsismo lógico y práctico. En cambio, una vez adoptado el punto de vista del hablante, dichos recursos fructificaron, constituyendo por fin a la filosofía como ética.

Pero ahora se trata de una ética cuya realización exige la política, en el sentido en que toma forma en el ser social y comunitario. Así, la dimensión personal de la ética persiste, pero ya no en el silencio místico al que le es inaccesible la palabra, sino por el contrario, en la palabra misma, aligerada del

${ }^{39}$ En esa condición comunitaria de la naturaleza y el ejercicio del lenguaje se ubica un biato, el "salto en el vacío" o "el seguir la regla a ciegas" del que nos habla Wittgenstein en la sección 219 de Investigaciones filosóficas. Ver p. 211 de la edición citada. 
lastre perturbador con la que se la carga inevitablemente, por el solo hecho de ser un hablante del lenguaje. Del solipsismo ético al ethos comunitario, el pensamiento de Wittgenstein construye a la filosofía misma como una ética y, en este sentido, como un territorio para la práctica política. 